\title{
Ferramenta para geração e utilização de modelos de avaliação automática da qualidade de objetos de aprendizagem
}

\author{
Cristian Cechinel ${ }^{1}$, Ana Marilza Pernas², Vinícius Vieira dos Santos ${ }^{2}$ \\ ${ }^{1}$ Faculdade de Educação - Universidade Federal de Pelotas (UFPel) \\ Rua Benjamin Constant, 897 - CEP 96010-020 - Pelotas - RS - Brazil \\ contatodcristiancechinel.pro.br
}

${ }^{2}$ Centro de Desenvolvimento Tecnológico - Universidade Federal de Pelotas (UFPel) Campus Universitário s/n, Prédio 19 - Caixa Postal: 354 - 96010-900 - Pelotas - RS Brasil

marilza@inf.ufpel.edu.br, viniciussantos1924@gmail.com

\begin{abstract}
Assessing quality of learning objects (LO) is a difficult and complex task and the existing repositories normally adopt strategies for quality evaluation that rely on the opinions of their community of users. The existence of these contextual evaluations about LOs inside repositories can be used as the basis for comparison among those groups of resources considered good against those considered not-good. The present work presents a tool that identifies intrinsic features of LOs that can be associated to quality and that uses such metrics to generate models for automated quality assessment of LOs. The paper describes the process of development and the main functionalities of such tool that uses data collected from the MERLOT repository.
\end{abstract}

Resumo. A avaliação de qualidade de objetos de aprendizagem (OA) é uma tarefa complexa e os atuais repositórios normalmente utilizam estratégias de avaliação baseadas nas opiniões de sua comunidade de usuários. A disponibilização dessas avaliações contextuais a respeito dos OAs pode ser utilizada como base para a comparação de características de OAs considerados bons e não-bons por essa comunidade. O presente trabalho tem como objetivo apresentar uma ferramenta que identifica métricas intrínsecas aos OAs que possam estar associadas com a sua qualidade. Essas métricas são então utilizadas durante a geração de modelos de avaliação automática da qualidade. $O$ trabalho apresenta o desenvolvimento e as funcionalidades dessa ferramenta que utiliza dados de OAs recolhidos do repositório MERLOT.

\section{Introdução}

Avaliar a qualidade de objetos de aprendizagem (OA) é uma tarefa difícil e complexa que normalmente gira em torno de múltiplos e diferentes aspectos que precisam ser observados. Na verdade, a própria definição de qualidade não é direta. Vargo, Nesbit, 
Belfer \& Archambault (2003) declaram que, ainda que a avaliação da qualidade de OAs possa ser considerada um campo relativamente novo, ela tem suas origens em um extensivo campo de conhecimento anterior, relacionado à avaliação de software instrucional. Como declarado por Bethard, Wetzer, Butcher, Martin \& Sumner (2009), a qualidade é contextual e irá depender do "alinhamento entre o grupo de usuário sendo servidos pelo objeto, a ambientação em que o objeto foi implementado, e o propósito designado para o objeto".

Após a sua produção, os OAs precisam ser publicados em um lugar onde os usuários possam buscar e recuperar os mesmos para uso futuro, uma fase definida dentro do ciclo de vida de um OA como oferta (2004). Os Repositórios de OAs (LORs) são os sistemas que fornecem as funcionalidades para isso, se tornando potenciais agregadores de comunidades de prática (Brosnan, 2005; Han, Kortemeyer, Krämer, \& von Prümmer, 2008), i.e. pessoas que compartilham interesses e preocupações sobre alguma coisa que eles fazem e que aprendem por meio das interações entre eles. Por conta disso, os LORs tendem a aproveitar as características de tais ambientes sociais e adotam estratégias para o estabelecimento da qualidade, contando com as impressões de uso e as avaliações dadas pelos usuários e especialistas membros da comunidade do repositório. Entre outros fatores, essas estratégias se sustentam: no valor dos metadados desde a perspectiva da teoria do capital social, i.e., que permite e fortalece relações sociais que possuem o potencial para possibilitar o acúmulo de benefícios para os indivíduos, sejam eles econômicos ou não (Lytras, Sicilia, \& Cechinel, 2014).

Vuorikari et al. (2008) denominam esse tipo de informação de metadados avaliativos. De acordo com os autores, "o metadado avaliativo possui uma natureza acumulativa, significando que as anotações dos diferentes usuários acumulam ao longo do tempo, em oposição a ter uma única avaliação autoritária". Dentro dos LORs, as informações avaliativas são normalmente utilizadas como a base para assegurar a qualidade dos recursos, mas também para o ranqueamento e a recomendação dos mesmos para os usuários.

Ainda que as estratégias atuais para a avaliação dentro de repositórios possam ser consideradas bem sucedidas até certo ponto, a quantidade de OAs dentro dos mesmos tende a crescer mais rapidamente do que a capacidade de suas comunidades avaliarem esses recursos (Cechinel \& Sánchez-Alonso, 2011). Tal condição torna impraticável confiar somente no esforço humano para classificar recursos de boa qualidade, na medida em que se torna impossível fornecer metadados avaliativos para cada novo recurso do repositório.

Esse problema tem levantado a preocupação com o desenvolvimento de novas técnicas e ferramentas automáticas e que possam ser utilizadas para complementar as abordagens existentes de maneira a aliviar o trabalho manual. A atual abundância de recursos dentro dos repositórios (Ochoa \& Duval, 2009) e a disponibilidade de avaliações contextuais em muitos deles abre a possibilidade de buscar por métricas intrínsecas dos OAs que possam ser utilizadas como indicadores de qualidade. Neste sentido, o presente trabalho tem o objetivo de automatizar parte do processo de avaliação de qualidade, oferecendo uma ferramenta capaz de minerar métricas quantitativas de recursos bons e não-bons, de forma a compará-las para a descoberta de atributos intrínsecos associado com a qualidade, permitindo assim a criação de perfis 
estatísticos de bons e não-bons recursos que podem servir como base para a predição da qualidade.

Mesmo sabendo que a análise automática não pode substituir as técnicas de inspeção tradicionais, ela possui o potencial de oferecer um mecanismo barato e de economia de tempo para explorar de antemão a qualidade dos materiais, complementando, portanto, outras abordagens existentes. A existência de uma ferramenta para automatizar a identificação destes atributos pode melhorar a qualidade dos serviços fornecidos pelos repositórios com relação aos processos de busca, seleção e recomendação dos materiais de boa qualidade. Contribuidores poderiam, por exemplo, se beneficiar de tal característica avaliando antecipadamente a qualidade de seus recursos, o que lhes permitiria o melhoramento dos mesmos através do uso das métricas de qualidade referenciadas pela ferramenta.

O restante desse trabalho é estruturado da seguinte maneira. A seção 2 apresenta o estado da arte e os trabalhos que foram utilizados como base para a implementação da ferramenta descrita. A seção 3 descreve o desenvolvimento da ferramenta e sua utilização. Na seção 4 são apresentadas as considerações finais e possibilidades de trabalhos futuros.

\section{Estado da arte}

Trabalhos voltados para avaliação automática de OAs têm sido desenvolvidos por Cechinel, Sánchez-Alonso, and García-Barriocanal (2011), que propuseram uma abordagem complementar para avaliação automática da qualidade que utiliza somente dados que podem ser diretamente extraídos dos próprios recursos em combinação com os metadados avaliativos do repositório. A principal vantagem da proposta é oferecer uma ferramenta capaz de medir a qualidade de novos recursos inseridos no repositório sem a necessidade de anotações (pontuações, metadados avaliativos) sobre os mesmos. Os autores forneceram os fundamentos para o desenvolvimento de tal ferramenta, a partir do contraste de métricas intrínsecas de OAs altamente pontuados e pobremente pontuados e armazenados no repositório MERLOT (www.merlot.org), além da identificação de quais métricas estão mais associadas com as pontuações naquele repositório. Nesse trabalho, os autores descobriram que as métricas testadas apresentam diferentes perfis e tendências entre materiais bons e não-bons, dependendo da categoria de disciplina e do tipo de material ao qual o recurso pertence. Por exemplo, foram encontradas correlações positivas entre a qualidade dos OAs e o Número de Imagens para recursos das disciplinas de Educação, Matemática e Estatística, e de Ciência e Tecnologia; e entre o Número de Applets e a disciplina de Negócios (Cechinel, SánchezAlonso, \& García-Barriocanal, 2011). Ainda, os autores construíram um modelo utilizando Análise Discriminante Linear com base nessas métricas, o que foi capaz de distinguir entre bons e não-bons OAs (para a disciplina de Ciência e Tecnologia e do tipo Simulação) com uma precisão geral de 91,49\%, um ótimo resultado para uma tentativa preliminar com foco na avaliação automática.

Em outro experimento, Cechinel, da Silva Camargo, Ochoa, Sicilia, and Sanchez-Alonso (2012) testaram uma abordagem levemente diferente e mais algorítmica, i.e., os modelos foram gerados exclusivamente por meio do uso de algoritmos de mineração de dados. Entre outros bons resultados, pode-se mencionar o 
modelo para a disciplina de Humanidades e o tipo de material Simulação (Humanidades $\cap$ Simulação) que foi capaz de classificar recursos bons com $75 \%$ de precisão e nãobons com 79\%; além do modelo desenvolvido para Matemática $\cap$ Tutorial com 79\% de precisão para classificar recursos bons e 64\% para classificar recursos não-bons.

A mesma abordagem foi testada para o repositório Connexions (www.cnx.org) em Cechinel, Sánchez-Alonso, Sicilia, and Simões (2011), entretanto os modelos apresentaram desempenhos ruins para classificar os recursos. De acordo com os autores, isso pode ser uma consequência do tamanho pequeno da população de recursos que possuíam metadados avaliativos (lenses). Por conta disso, é ainda necessário esperar o crescimento dessas informações dentro do repositório para que se possa avaliar melhor a viabilidade de criar modelos para avaliar automaticamente a qualidade dos recursos de acordo com o número de lentes. Nos trabalhos mencionados, os autores utilizaram informações (categorias de disciplinas, tipos de materiais, avaliações de especialistas e de usuários, lentes) que não estão necessariamente disponíveis em todos os LORs. Nesses casos onde algumas dessas informações não estejam disponíveis, maneiras alternativas de buscar pela qualidade dos OAs precisam ser encontradas para poder contrastar com as métricas e o consequente estabelecimento dos perfis, tais como, por exemplo, o uso de métricas de ranqueamento (Ochoa \& Duval, 2008) ou outros tipos de metadados avaliativos disponíveis em tais repositórios.

\section{Desenvolvimento da ferramenta}

A ferramenta foi desenvolvida em linguagem Java, com base na metodologia descrita em Cechinel, Sánchez-Alonso, and García-Barriocanal (2011). Sua visão geral éapresentada na Figura 1.

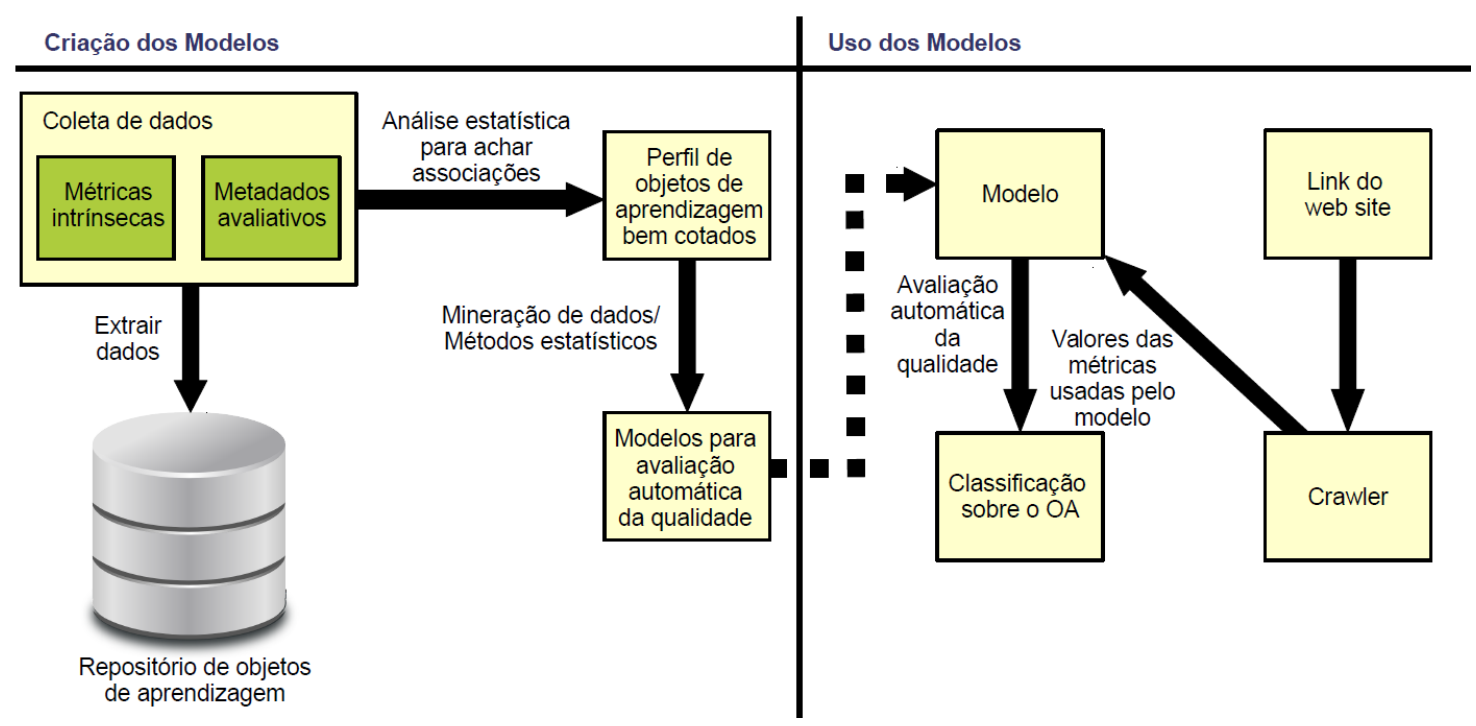

Figura 1. Visão geral da metodologia implementada na ferramenta

Para o desenvolvimento a base de dados de um repositório (nesse caso, o repositório MERLOT) foi recolhida por meio de um crawler que coletou informações sobre os OAs, tais como: pontuações fornecidas pela comunidade de usuários e pela comunidade de especialistas do repositório, classificação dos OAs com relação ao tipo de material (Animação, Simulação, Tutorial, etc) e a disciplina (Artes, Negócios, 
Educação, Humanas, Matemática e Estatística, Ciência e Tecnologia, e Ciências Sociais). Além dessas informações, foram recolhidas 34 métricas intrínsecas dos objetos que são avaliadas como possíveis indicadores de qualidade (veja Tabela 1).

Tabela 1. Métricas Utilizadas no Estudo

\begin{tabular}{|l|l|}
\hline \multicolumn{1}{|c|}{ Classe da Métrica } & \multicolumn{1}{|c|}{ Métrica } \\
\hline Medidas de Link & $\begin{array}{l}\text { Número de Links, Número de Links Únicos, Número de Links } \\
\text { Internos, Número de Links Internos Únicos, Número de Links } \\
\text { Externos, Número de Links Externos Únicos. }\end{array}$ \\
\hline Medidas de Texto & Número de Palavras, Número de Palavras que são Links. \\
\hline $\begin{array}{l}\text { Medidas Gráficas, de } \\
\text { Interatividade e } \\
\text { Multimídia }\end{array}$ & $\begin{array}{l}\text { Número de Imagens, Tamanho Total das Imagens (em bytes), } \\
\text { Número de Scripts, Número de Applets, Número de Arquivos de } \\
\text { Áudio, Número de Arquivos de Vídeo, Número de Arquivos } \\
\text { Multimídia. }\end{array}$ \\
\hline $\begin{array}{l}\text { Medidas de } \\
\text { Arquitetura do } \\
\text { Website }\end{array}$ & $\begin{array}{l}\text { Tamanho da Página (em bytes), Número de Arquivos para Download, } \\
\text { Número Total de Páginas. }\end{array}$ \\
\hline
\end{tabular}

Os OAs pertencentes aos diversos subconjuntos existentes (tipo de material versus categoria de disciplina) são divididos em classes de qualidade de acordo com as informações avaliativas disponíveis sobre os mesmos (avaliações de usuários, avaliações de especialistas e inclusão dos objetos em bookmarks de usuários). Essas classes de qualidade são então contrastadas entre si considerando cada uma das métricas intrínsecas coletadas. Sempre que a métrica avaliada apresenta uma diferença estatística significativa entre as classes, é considerada como um possível indicador de qualidade.

As análises estatísticas realizadas para a avaliação das diferenças entre as amostras de cada uma das métricas foram conduzidas da seguinte maneira. Como as amostras não seguem uma distribuição normal, um teste de Mann-Whitney (Wilcoxon) foi executado para avaliar se as classes apresentam diferenças entre suas medianas, e um teste de Kolmogorov-Smirnov foi aplicado para avaliar se as classes apresentam distribuições diferentes. Quando ambas (distribuições e medianas) apresentam diferenças significativas, a métrica é então considerada como um potencial indicador de qualidade. A tendência de cada métrica (se a mesma influencia positivamente ou negativamente a qualidade do OA) foi observada a partir da comparação entre as medianas dos valores das amostras. As métricas identificadas como possíveis indicadores de qualidade são então sugeridas ao usuário para servirem como entrada para os algoritmos de mineração, os quais irão gerar os modelos de avaliação de qualidade.

Os modelos para avaliação de qualidade gerados podem ser salvos e reutilizados para a avaliação de um novo OA disponível na web. É importante ressaltar que os OAs disponibilizados no MERLOT são em sua grande parte constituídos de páginas web, sendo que a ferramenta desenvolvida também trabalha com OAs nesse formato. As limitações dessa abordagem foram apontadas nos trabalhos anteriores já citados.

\subsection{Demonstração de funcionamento}

Um arquivo de configuração contém as informações para o acesso ao banco de dados criado pelo webcrawler. Após a conexão com o banco de dados o mesmo é carregado na 
interface da ferramenta, que apresenta as informações gerais sobre os conjuntos de OAs existentes, sendo elas: categorias de disciplinas, tipos de materiais, números de objetos que possuem avaliações de acordo com as perspectivas dos usuários e dos especialistas, e número de coleções pessoais ${ }^{1}$ em que os OAs estão inseridos.

A Figura 2 apresenta a tela inicial da ferramenta onde são apresentadas as informações gerais sobre os OAs. Como pode ser observado na figura, o usuário pode escolher a perspectiva de qualidade a ser observada para gerar os modelos, assim como a categoria de disciplina e o tipo de material. No exemplo em questão são selecionadas a perspectiva de qualidade dos especialistas e o conjunto dos OAs da disciplina Ciência e Tecnologia versus tipo de material Tutorial (parte esquerda). Ao lado esquerdo da figura, são apresentadas as quantidades de objetos pertencentes ao conjunto e os valores de notas que definem as classes de qualidade bom e não-bom dos objetos. Para o exemplo em questão, os materiais bons são aqueles que possuem notas dadas pelos especialistas entre 4,67 e 5 (região azul do gráfico), e os não bons são aqueles com notas de 4,66 para baixo (região rosa do gráfico).

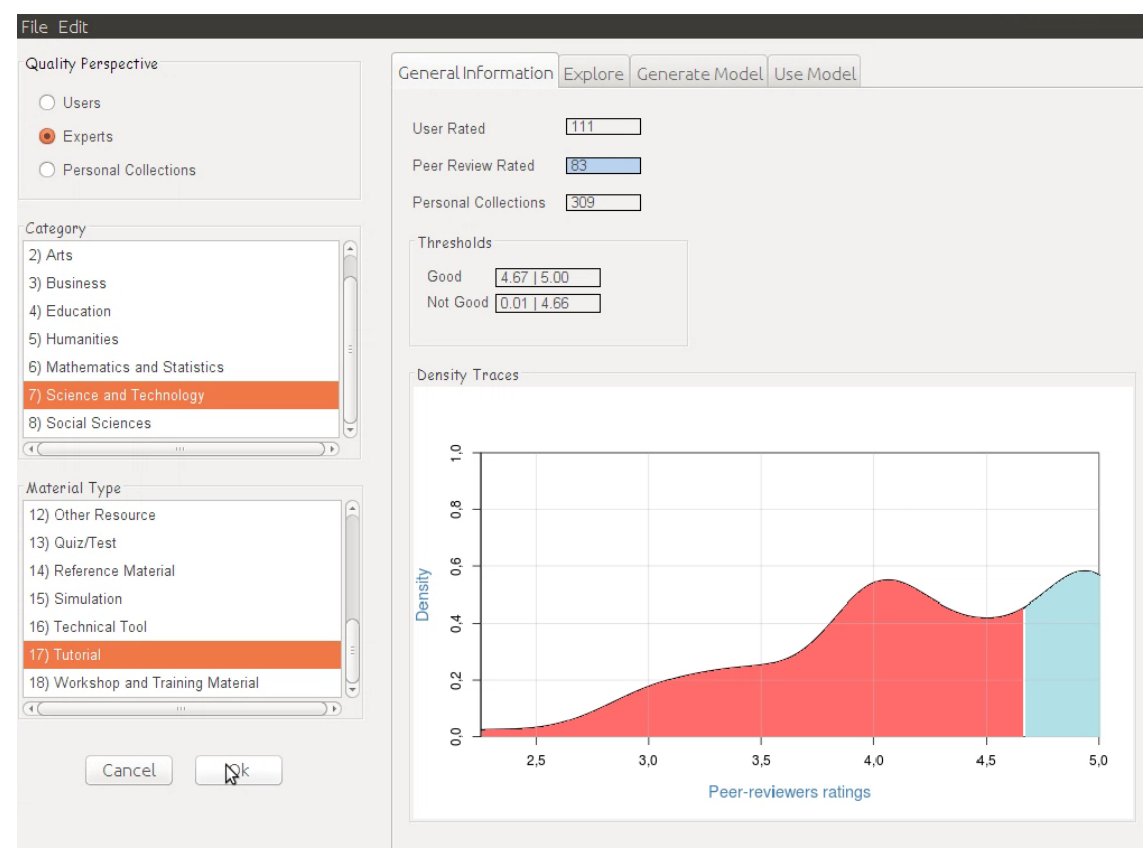

Figura 2. Tela Inicial - Informações Gerais

Após selecionar o conjunto e clicar em $\mathrm{OK}$, são apresentadas para o usuário as métricas que apresentaram diferenças estatisticamente significantes entre os conjuntos de OAs bons e não-bons a partir das análises de Mann-Whitney e de Kolmogorov. Todas as análises estatísticas e os gráficos gerados foram realizados utilizando scripts do pacote estatístico $\mathrm{R}^{2}$. A Figura 3 demonstra um exemplo de apresentação das métricas indicadas. Como pode ser visualizado na figura, as métricas aparecem marcadas em amarelo e pré-selecionadas para utilização na geração do modelo de avaliação. Também é apresentada a influência da métrica na qualidade do OA, ou seja,

${ }^{1}$ O MERLOT permite que seus usuários adicionem os recursos em coleções pessoais, fornecendo uma maneira dos mesmos organizarem seus materiais favoritos de acordo com seus interesses individuais.

${ }^{2}$ http://www.r-project.org/ 
se ela influencia positivamente ou negativamente. No exemplo em questão, o número de imagens (Links_Number) aparece com uma seta para baixo, indicando que quanto maior o número de imagens para aquele tipo de $\mathrm{OA}$, menor é qualidade do mesmo (de acordo com as análises estatísticas realizadas). Por último, algumas estatísticas descritivas e os resultados dos testes de Mann-Whitney e Kolmogorov também são apresentados.

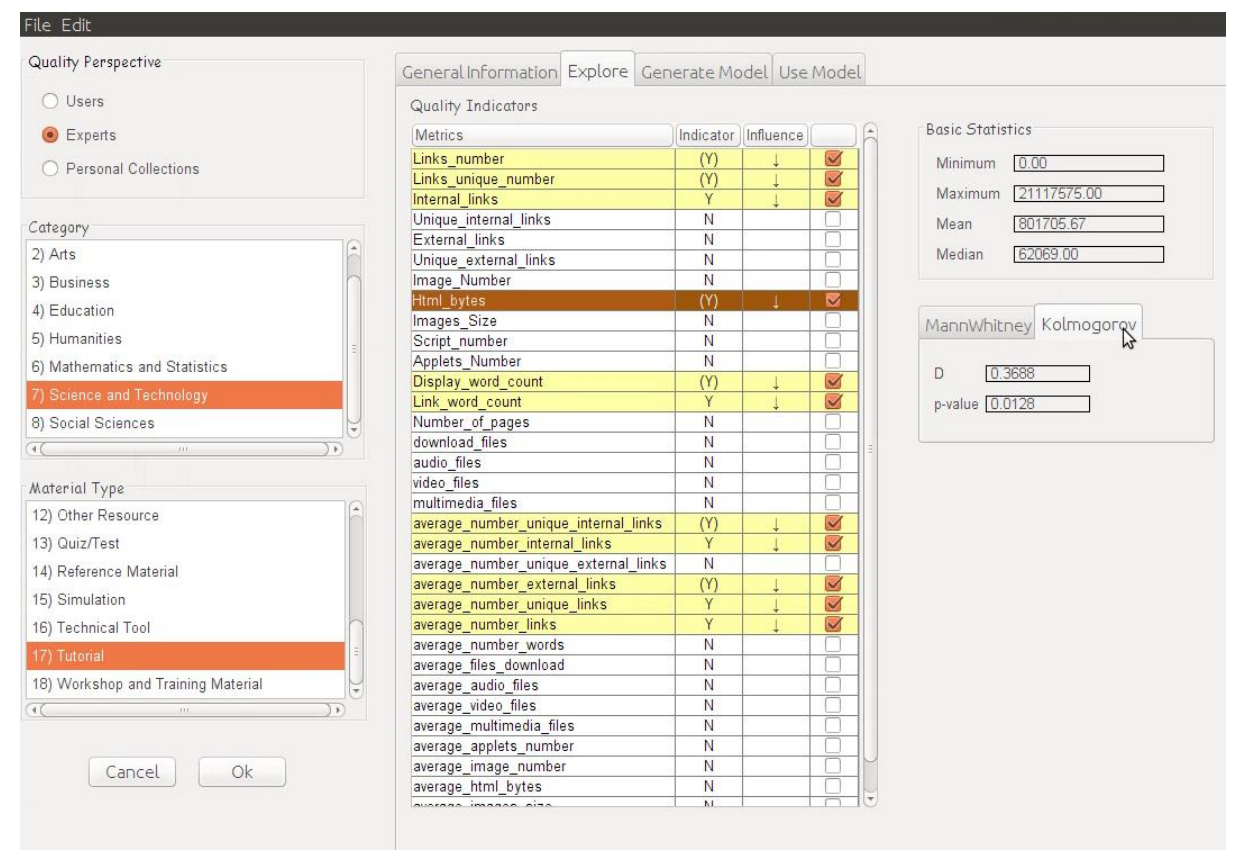

Figura 3. Visualização das métricas recomendadas

Após a solicitação para gerar o modelo de avaliação, a ferramenta executa o algoritmo J48 implementado no $\mathrm{WEKA}^{3}$ e apresenta ao usuário a árvore gerada e as medidas de avaliação encontradas para essa árvore. Nesta versão da ferramenta apenas o algoritmo J48 foi implementado, porém outras técnicas de mineração serão incorporadas nas próximas versões. No exemplo apresentado na Figura 4 é possível visualizar uma árvore com seis folhas (janela da esquerda - Model), e com precisão de $89 \%$ para a classificação dos OAs bons e $82,8 \%$ para a classificação dos OAs não bons (janela da direita - Evaluation). No canto superior esquerdo existe a opção de salvar o modelo gerado para que possa ser posteriormente utilizado na avaliação de outro OA.

Como comentado, o modelo pode agora ser gerado para avaliar um OA qualquer disponível na internet, sendo que todas as características e métricas utilizadas nessa avaliação podem ser observadas pelo usuário. Como pode ser visto na Figura 5, para avaliar um OA o usuário deve digitar o endereço web do mesmo, solicitar para que a ferramenta calcule as métricas intrínsecas desse OA (botão Get Metrics) e a sua classificação entre as classes Bom e Não-Bom (Classify Data). O cálculo das métricas é realizado pelo mesmo webcrawler utilizado para recolher as informações do banco de dados utilizado na pesquisa. A ferramenta irá apresentar as informações coletadas e a avaliação gerada pelo modelo para esse OA. É importante ressaltar que o OA deve pertencer a mesma categoria de disciplina e tipo de material para o qual o modelo de

\footnotetext{
${ }^{3}$ www.cs.waikato.ac.nz/ml/weka/
} 
avaliação foi gerado para que a avaliação automática seja coerente com a proposta metodológica.

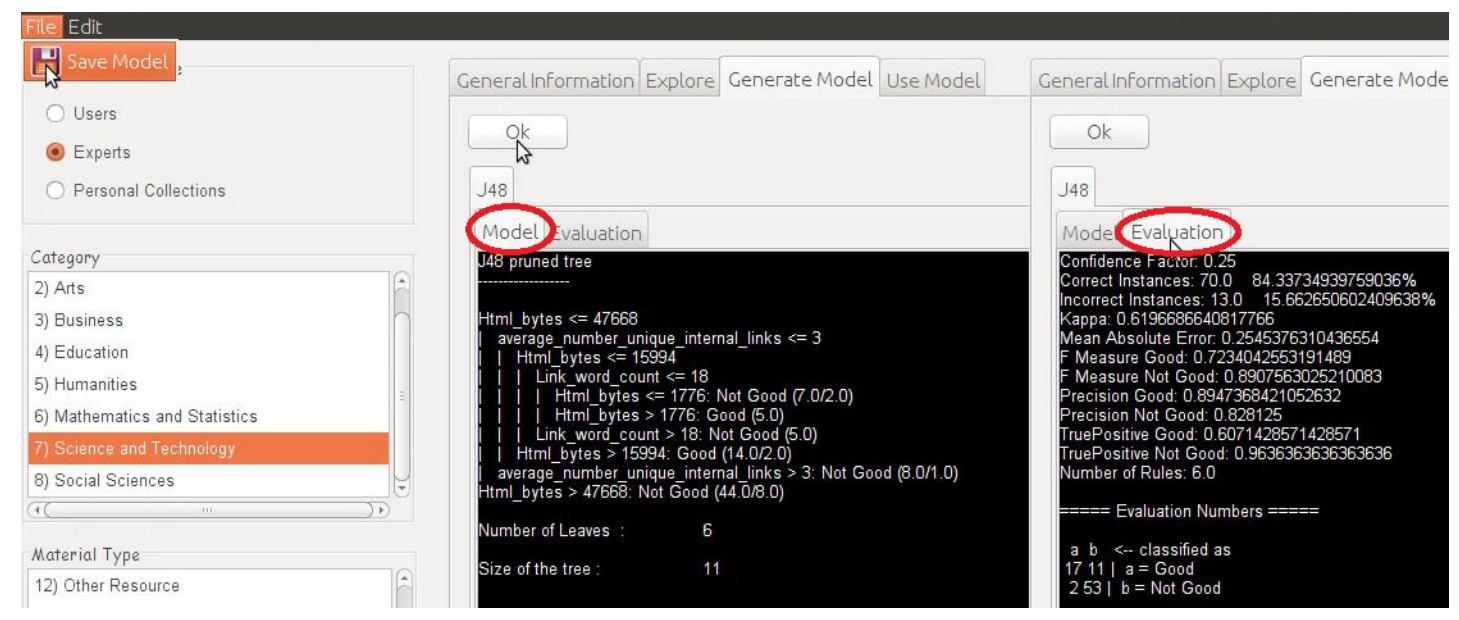

Figura 4. Apresentação da árvore e gerada pelo J48 (janela esquerda) e sua avaliação (janela direita)

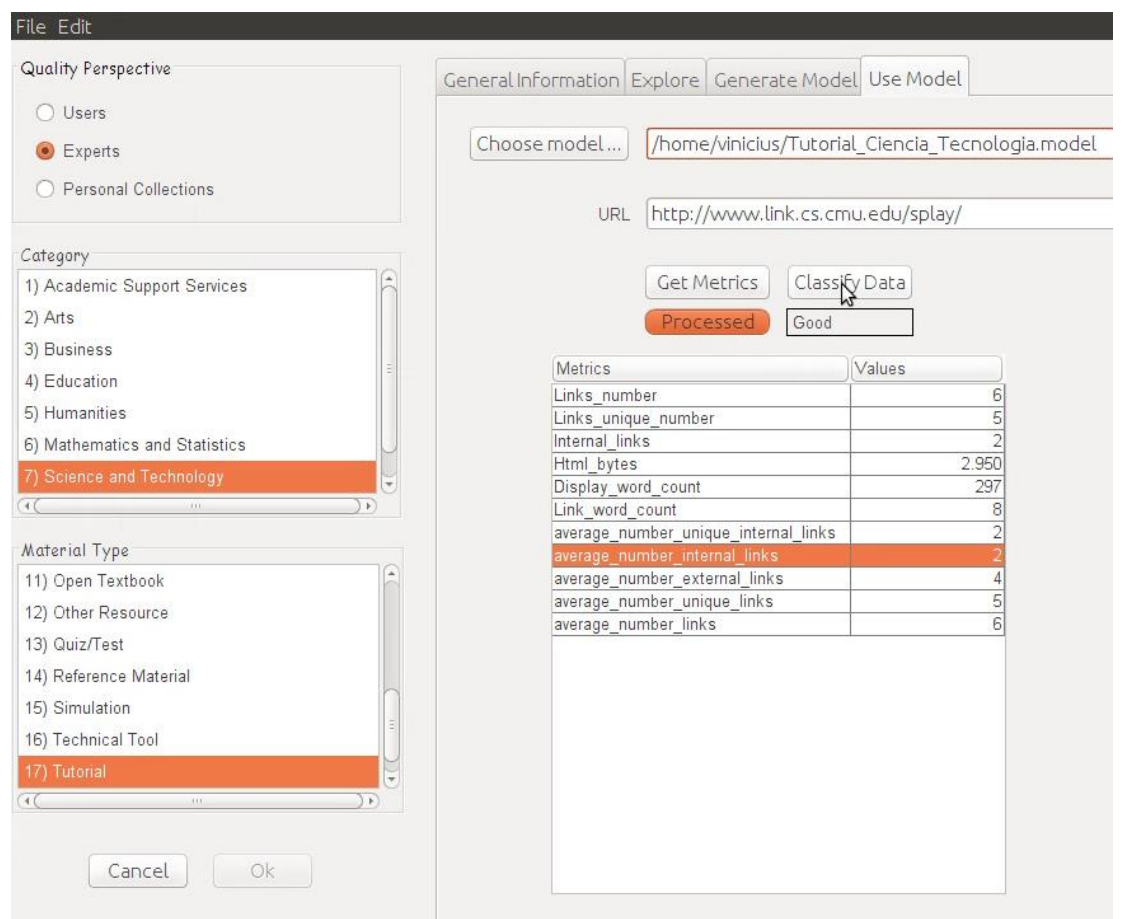

Figura 5. Utilizando o modelo para avaliar um OA

\section{Considerações finais}

A avaliação da qualidade dos objetos de aprendizagem é uma tarefa difícil que normalmente envolve diversos aspectos distintos e diferentes atores interessados, e os métodos de avaliação atualmente existentes não estão livres de ambiguidades. Diferentes repositórios de objetos de aprendizagem estão frequentemente adotando 
estratégias que confiam na comunidade de usuários e especialistas que avaliam a qualidade dos recursos a partir de pontos e comentários. Tais avaliações podem ser realizadas a partir de critérios de avaliação formais e pré-definidos, ou de maneira mais informal e sem especificações pré-estabelecidas. O conjunto de avaliações resultantes é então utilizado pelos LORs para facilitar o processo de busca e ranqueamento dos OAs e é considerado como um corpo de conhecimento social que serve como uma memória externa de ajuda para os indivíduos que navegam em tais portais. Ao mesmo tempo em que as estratégias atuais se estabeleceram como a principal alternativa para a avaliação da qualidade dentro de LORs, elas ainda são insuficientes para cobrir a enorme quantidade de recursos que continua crescendo em tais plataformas. Portanto, existe uma necessidade urgente do desenvolvimento de alternativas que possam ajudar a estimular o abastecimento de informações sobre a qualidade em complemento as estratégias manuais atualmente existentes. A ferramenta desenvolvida e apresentada nesse trabalho é um primeiro passo nessa direção.

Os modelos de avaliação automática gerados pela ferramenta podem conter informações úteis para os criadores que desejam ter uma avaliação preliminar de seus materiais com o objetivo de melhorá-los. Durante a fase de obtenção (ou criação) (Collis \& Strijker, 2004) do OA, os autores podem ter acesso a uma avaliação preliminar da qualidade do mesmo com relação às características intrínsecas incluídas nesses modelos. A utilização de um modelo em árvore (J48) permite a explicação do raciocínio percorrido para chegar aos resultados da classificação. Uma vez que a avaliação preliminar é realizada, o criador pode consultar o modelo (e os perfis estatísticos dos recursos) para entender quais medidas intrínsecas do OA estão influenciando essa classificação e melhorar seu OA de acordo com essas medidas. Trabalhos futuros irão incorporar novos algoritmos de mineração de dados dentro da ferramenta, assim como estudar a viabilidade de utilização da mesma para bancos de dados extraídos de outros repositórios.

\section{Agradecimentos}

Esse trabalho foi financiado pelo CYTED (Programa Ibero-Americano de Ciência e Tecnologia para o Desenvolvimento) como parte do projeto "RIURE - Rede IberoAmericana para a Usabilidade de Repositórios de Aprendizagem“ (código 513RT0471) - www.riure.net .

\section{Referências}

Bethard, S., Wetzer, P., Butcher, K., Martin, J. H., \& Sumner, T. (2009). Automatically characterizing resource quality for educational digital libraries. Paper presented at the Proceedings of the 9th ACM/IEEE-CS joint conference on Digital libraries, Austin.

Brosnan, K. (2005). Developing and sustaining a national learning-object sharing network: A social capital theory perspective. Paper presented at the Proceedings of the ASCILITE 2005 Conference, Brisbane, Australia.

Cechinel, C., da Silva Camargo, S., Ochoa, X., Sicilia, M., \& Sanchez-Alonso, S. (2012). Populating learning object repositories with hidden internal quality 
information. Paper presented at the 2nd Workshop on Recommender Systems for Technology Enhanced Learning (RecSysTEL), Saarbrücken.

Cechinel, C., \& Sánchez-Alonso, S. (2011). Analyzing Associations between the Different Ratings Dimensions of the MERLOT Repository. Interdisciplinary Journal of E-Learning and Learning Objects 7, 1-9.

Cechinel, C., Sánchez-Alonso, S., \& García-Barriocanal, E. (2011). Statistical profiles of highly-rated learning objects. Computers \& Education, 57(1), 1255-1269. doi: 10.1016/j.compedu.2011.01.012

Cechinel, C., Sánchez-Alonso, S., Sicilia, M.-Á., \& Simões, P. W. T. d. A. (2011). Exploring the Development of Endorsed Learning Resources Profiles in the Connexions Repository. In E. García-Barriocanal, Z. Cebeci, M. C. Okur \& A. Öztürk (Eds.), Metadata and Semantic Research (Vol. 240, pp. 12-21): Springer Berlin Heidelberg.

Collis, B., \& Strijker, A. (2004). Technology and Human Issues in Reusing Learning Objects. Journal of Interactive Media in Education; May 2004: JIME Special Issue on the Educational Semantic Web, 2004(1).

Han, P., Kortemeyer, G., Krämer, B., \& von Prümmer, C. (2008). Exposure and Support of Latent Social Networks among Learning Object Repository Users. Journal of the Universal Computer Science, 14(10), 1717-1738. doi: citeulike-articleid:3558788

Lytras, M. D., Sicilia, M.-Á., \& Cechinel, C. (2014). The value and cost of metadata. In M.-Á. Sicilia (Ed.), Handbook of Metadata, Semantics and Ontologies (pp. 4162): World Scientific Publishing Company.

Ochoa, X., \& Duval, E. (2008). Relevance Ranking Metrics for Learning Objects. Learning Technologies, IEEE Transactions on, 1(1), 34-48. doi: http://dx.doi.org/10.1109/TLT.2008.1

Ochoa, X., \& Duval, E. (2009). Quantitative Analysis of Learning Object Repositories. Learning Technologies, IEEE Transactions on, 2(3), 226-238.

Vargo, J., Nesbit, J. C., Belfer, K., \& Archambault, A. (2003). Learning Object Evaluation: Computer-Mediated Collaboration and Inter-Rater Reliability. International Journal of Computers and Applications, 25(3), 1-8.

Vuorikari, R., Manouselis, N., \& Duval, E. (2008). Using Metadata for Storing, Sharing and Reusing Evaluations for Social Recommendations: the Case of Learning Resources. Social Information Retrieval Systems: Emerging Technologies and Applications for Searching the Web Effectively. Hershey, PA: Idea Group Publishing, 87-107. 